\title{
EBM 2010
}

\section{Nicht in die Budgetfalle tappen!}

\author{
Budgets dienen der Kassenärztlichen Bundesvereinigung (KBV) und den \\ Kassenärztlichen Vereinigungen der Länder sowie den Krankenkassen als \\ Mengenbegrenzung. Für den Vertragsarzt können diese Budgets ab 1. Juli \\ 2010 aber zur Falle werden.
}

— Die schöne neue Welt der Hausarztverträge induziert eine wünschenswerte Arbeitsweise, die außerhalb der IV-Verträge zu einem spürbaren Verlust führen kann. In den IV-Verträgen erhält der Vertragsarzt eine Vergütung für seine Qualifikationen und die vorgehaltenen technischen Möglichkeiten. Leistungen erbringt er immer dann, wenn das Krankheitsbild und der Verlauf der Krankheit eine bestimmte Diagnostik und Therapie erfordern: Leistungserbringung aufgrund bestehender Leitlinien und Indikation.

\section{Leistungsvolumina müssen „abverdient" werden}

In der Vertragswelt der KV gibt es Leistungsvolumen, die erst „abverdient" werden müssen. Die derzeit noch gel- tenden Zusatzvolumina für Sonografie, Spirometrie, Belastungs-EKG usw. sind ein Beispiel dafür. Erbringt ein Vertragsarzt in diesem Bereich nicht entsprechende Leistungsmengen, geht Umsatz verloren. Der Verlust gefährdet die wirtschaftliche Praxisführung und reduziert das Einkommen. Hier werden die Indikation und die Therapie zwangsweise von wirtschaftlichen Überlegungen mitbestimmt.

Die Arztpraxis als Wirtschaftsbetrieb erfordert es aber, dass die zur Verfügung stehenden Budgets ausgeschöpft werden. Eine schwierige Balance, denn häufig ist die Klientel der Praxis von Quartal zu Quartal bezüglich der Morbidität sehr unterschiedlich. Wirtschaftlich ideal steht der Vertragsarzt da, der es schafft, eine Punkt- landung zu erreichen: In diesem Fall schöpft er die ihm zur Verfügung stehenden Budgets aus, vermeidet aber eine Überschreitung. Im Fall der Überschreitung reicht die Vergütung der "Restpunkte" in der Regel noch nicht einmal zur Kostendeckung. Dies führt ebenso zu einem Gewinn- und damit Einkommensverlust.

\section{Einmal keine Leistung erbracht, schon ist das QZZV weg}

Ab 1. Juli 2010 muss sich der Vertragsarzt die neuen „Spielregeln“ täglich vor Augen halten. Mehr noch, er muss dann zusätzlich darauf achten, dass er in jedem Quartal in jedem der zur Verfügung stehenden Budgets für Qualifikationsgebundene Zusatzvolumen (QZV) zumindest einmal eine Leistung erbringt!

Tut er das nicht, verliert er das betreffende QZV im entsprechenden Quartal des Folgejahres. Es sei denn, die Abrechnungsmodalitäten sind dann schon wieder geändert.

\section{Seminarankündigung EBM 2010}

Unser Gebührenordnungsexperte Helmut Walbert macht Sie rechtzeitig fit mit Strategien zu Qualitätsbezogenen Zusatzvolumen (OZV), RLV, Budgets:

\section{Orte und Termine:}

Würzburg: 11. Mai 2010 Heidelberg: 19. Mai 2010 Köln: $\quad 9 . J u n i 2010$ Hannover: 30. Juni 2010

$$
\begin{array}{lr}
\text { Hamburg: } & \text { 2. Juli } 2010 \\
\text { Berlin: } & \text { 3. Juli } 2010 \\
\text { Nürnberg: } & \text { 7. Juli } 2010 \\
\text { München: } & \text { 10. Juli } 2010
\end{array}
$$

Nähere Infos unter:

Tel.: 09312998594

E-Mail:w@lbert.info

Fax: 093125090601

Website: www.walbert-helmut.de
Kommentar

\section{Ärztliche Ethik kontra Monetik}

Der niedergelassene Vertragsarzt sitzt in der Falle: zwei verschiedene Vergütungsmodelle zur gleichen Zeit. Das ist perfide, vor allem deswegen, weil zwei verschiedene Vergütungsansätze, IV-Verträge versus Budgetmodell der KVen, es theoretisch erfordern, dass ärztliches Handeln permanent - von Patient zu Patient - von wirtschaftlichem Denken gesteuert wird. Noch ist ärztliches Denken und Handeln aber überwiegend patientenorientiert. Ein Spagat, der im Alltag eines Hausarztes nicht durchzuhalten ist und damit im KV-System zulasten des Vertragsarztes ausgehen muss: Entweder er erreicht seine Budgets nicht oder er überschreitet sie. Das bedeutet in jedem Fall Gewinnverlust. In diesem Bewusstsein zu arbeiten, fördert keineswegs die Lust an der täglichen Arbeit: Ein weiteres Plus für IV-Verträge, wie sie beispielhaft der Bayerische Hausarztverband mit der AOK Bayern abgeschlossen hat.

H. WALBERT 